\title{
BASICS OF THEORETICAL APPROACHES OF TECHNOLOGICAL PROCESSES WITH REGULAR TOSSING OF THE GRANULAR MEDIUM
}

\author{
A.V. Eliseev ${ }^{a}$, A.I. Artyunin ${ }^{b}$, S.K. Kargapolcev and A.V. Dimov \\ 1 Irkutsk State Transport University, Irkutsk, Russia Federation

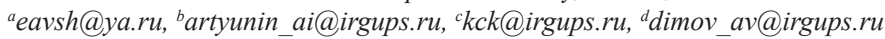

\begin{abstract}
Vibration interaction is used for development of modern technological processes in which required quality of product is provided due to interaction of the granular medium with the vibrating surface. The paper is devoted to development of mathematical models and estimation criteria form of movement, taking into account the effect of unilateral constraints, in dynamic interactions of elements of vibration technological processes as applied to increase the reliability and quality of vibrational machines. The study is focused on the formation of theoretical ideas about the dynamic interactions of a solid body with the vibrating surface based on the development of mathematical models by introducing of additional external forces and elastic ties with unilateral contact, including mathematical models of gapless movement of the compounded solids. In terms of practical applications the task is to develop and to approve experimentally the opportunities of the measuring devices fixing the modes of continuous tossing. According to the research the generalized approach to problems of dynamic synthesis of vibration technology implemented in continuous tossing the introduction of additional forces is developed. The basis of the approach is the introduction of the concept of the function of the gap, allowing to justify the construction of forms of motion of material particles in accordance with certain criteria, reflecting the properties of the trajectories of motion of material particles in the interaction with the vibrating surface. The mathematical models to determine the conditions for the formation of dynamic reactions in the vibrational structure consisting of several interconnected elements, including the possibilities of their pre-pressing by elastic ties and power factors is offered. The measuring device fixing the modes of continuous tossing is developed and experimentally approved.
\end{abstract}

Keyboards: unilateral constraints, the function of the gap, a composite solid, continuous tossing, vibration hardening, vibration interaction;

\section{INTRODUCTION}

Vibration technological processes were widely adopted in the production systems relating to various branches of equipment. Vibrations are not only have essential value in processes of vibrationmovement, transportation of objects and working environments, but vibrations are actively used in technologies of processing of details (vibration cutting of materials, vibration hardening, etc.) also. At the same time vibrations of mechanisms and machines impact on work of the equipment and devices negatively. Vibrations reduce 
reliability and durability of technical systems and create unsafe working conditions to the service personnel. In general, the area of manifestation of vibration interactions can be carried to rather developed direction of modern dynamics of machines. The theoretical basis of this direction is formed on a joint of theoretical mechanics, the theory of mechanisms and machines, the theory of fluctuations, the theory of vibration movement, the theory of vibration processes [19]. Considerable sections of the theory of vibration processes belong to area of nonlinear mechanics, the nonlinear theory of fluctuations and waves.

The attention to vibration movements is differentiated by consideration of separate problems of dynamics of machines. One of the most developed directions is the dynamic equilibration of machines and balancing of the rotors, initiation of vibration processes, problems of vibration protection of machines and equipment, etc. Vibration processes are various, as well as forms of influence on a dynamic condition of technical objects. Emergence of new constructive technical solutions, development of new classes of machines including robotic devices of different function initiates expansion of the front of researches. In this plan the processes of contacting of solid bodies, as standard elements of various mechanisms, with definition of conditions of formation of static and dynamic interactions are require urgent attention. In ideal cases such processes are studied as vibro-impact processes. It finds expression in development of the technical appendices connected with a surface treatment and increase of reliability of the details working in the conditions of intensive dynamic loading. In many cases vibration influence is considered as a factor of influence on a condition of the interacting parties of contact of the adjoining bodies and the connections arising thus. Features of unilateral connections which are characteristic for technological processes of vibro-movement, vibro-hardening, vibrotransportation etc. are less studied.

In the offered paper the possibility of development of the generalized approaches in the solution of problems of dynamic interactions of elements of technological systems with unilateral ties is considered.

\section{GENERAL PROVISIONS. STATEMENT OF RESEARCH PROBLEMS}

In recent years vibration interaction is rather often used for development of new technological processes in which the required quality of production is provided due to interaction of the loose environment with the vibrating surface. Examples of technical solutions of the features of vibroshock processes based for increase of physicomechanical properties of surfaces at impacts of small steel balls with details are reviewed in works [18].

Scientific works of R. F. Ganiyev, I.I. Blekhman, A.E. Kobrinsky, I.I. Bykhovsky, I.F. Goncharevich, V. I. Babitsky, etc. are well known as theoretical basis for vibration technological processes and vibrating machines.

Unilateral ties have a wide circulation in problems of dynamics of machines. Features of these connections are that under certain conditions, in particular at contact interactions when reaction is equal to zero, there can be changes of structure of initial mechanical system [10]. The change of system leads to emergence of additional effects.

Unilateral ties allow to realize processes of periodic impacts which are connected with opportunities of creation of vibrating technological processes.

Methods of mathematical modeling in problems of vibration interactions are well known. Such interactions are characteristic for technological processes of vibration movement, processes of vibration type which are used for processing of the contacting surfaces for the purpose of their hardening. It is 
shown that process of formation of interactions in systems with unilateral connections can be considered as the process consisting of several phases. Such phases are following: contact phases at which reaction of connection is positive; a phase of a transition state when reaction is equal to zero; a gap phase in which the contacting surfaces can make the autonomous movements.

Vibration technological machines (vibrating machines) which appointment consists in formation of a certain structure of a vibration field are used for justification of technological processes with continuous tossing. The scientific works in which theoretical bases of creation and maintenance of one-dimensional fluctuations develop have got most popularity [20].

In general, the problem of realization of vibration technological processes has complex character as technological machines are rather complex and, in turn, also contain knots and units in which unilateral ties there are between elements. Approaches to solution of the problem require the accounting of conditions of maintenance of steady vibration processes with tossing, and the simultaneous accounting of formation of the modes of dynamic interactions which are shown in reactions of connections and in contact interactions of compound structures of mechanisms and machines. Compound objects have some parts connected by the gravity or additional forces, and also elastic connections in which processes of contacts violation or considerable fluctuations of sizes of contact forces can be appear.

Research problems are focused on formation of theoretical ideas of dynamic interactions of a solid body with the vibrating surface on the basis of development of mathematical models at introduction of additional external forces and elastic connections at the registration of «not holding» contact, including, mathematical models of the gapless movement of compound bodies. In respect of practical applications the task of development and experimental approbation of opportunities of the measuring devices fixing the modes of continuous tossing with gap is set.

\section{MATHEMATICAL MODELING OF PROCESSES OF CONTINUOUS TOSSING WITH A GAP}

The generalized approach to an assessment of characteristics of trajectories of the movement of particles with formation of a phase of free approach with the subsequent interaction with the vibrating surface develops. As the basis the mathematical model of interaction of a material particle with the horizontal surface fluctuating under the harmonious law is used $H(t)=A \sin (\omega t)$ . Characteristics of interaction of a material particle on the vibrating surface considering of tuning parameters of basic and expanded mathematical models are determined. Expanded models reflect action of additional forces and viscous friction.

$$
\begin{aligned}
& R_{H}\left(t, t_{0}\right)=A \sin \left(\omega t_{0}\right)-A \sin (\omega t)+ \\
& +A \omega\left(t-t_{0}\right) \cos \left(\omega t_{0}\right)-\frac{1}{2} g\left(t-t_{0}\right)^{2}, t \geq t_{0}
\end{aligned}
$$

Basic elements of analytical approach are presented in tab. 1. Basic elements are following: basic model, connections with parameters, family of possible trajectories in a phase of flight (fig. 1), function of a gap, detachments condition. The concept of the function of a gap is introduced for comparison purposes opportunities of influence on process of adjusting parameters. The function of gap allows to conduct a detailed research of the most significant properties of vibration interactions.

For basic model with harmonious moving surface the function of a gap is following: 
The generalized approach on the basis of use of function of a gap assumes consideration of family of possible trajectories in a phase of free flight. Generally interaction of particles can happen to a surface at the laws of the movement of a surface having more complex form, than harmonious.

Table 1. Elements of the analytical approach

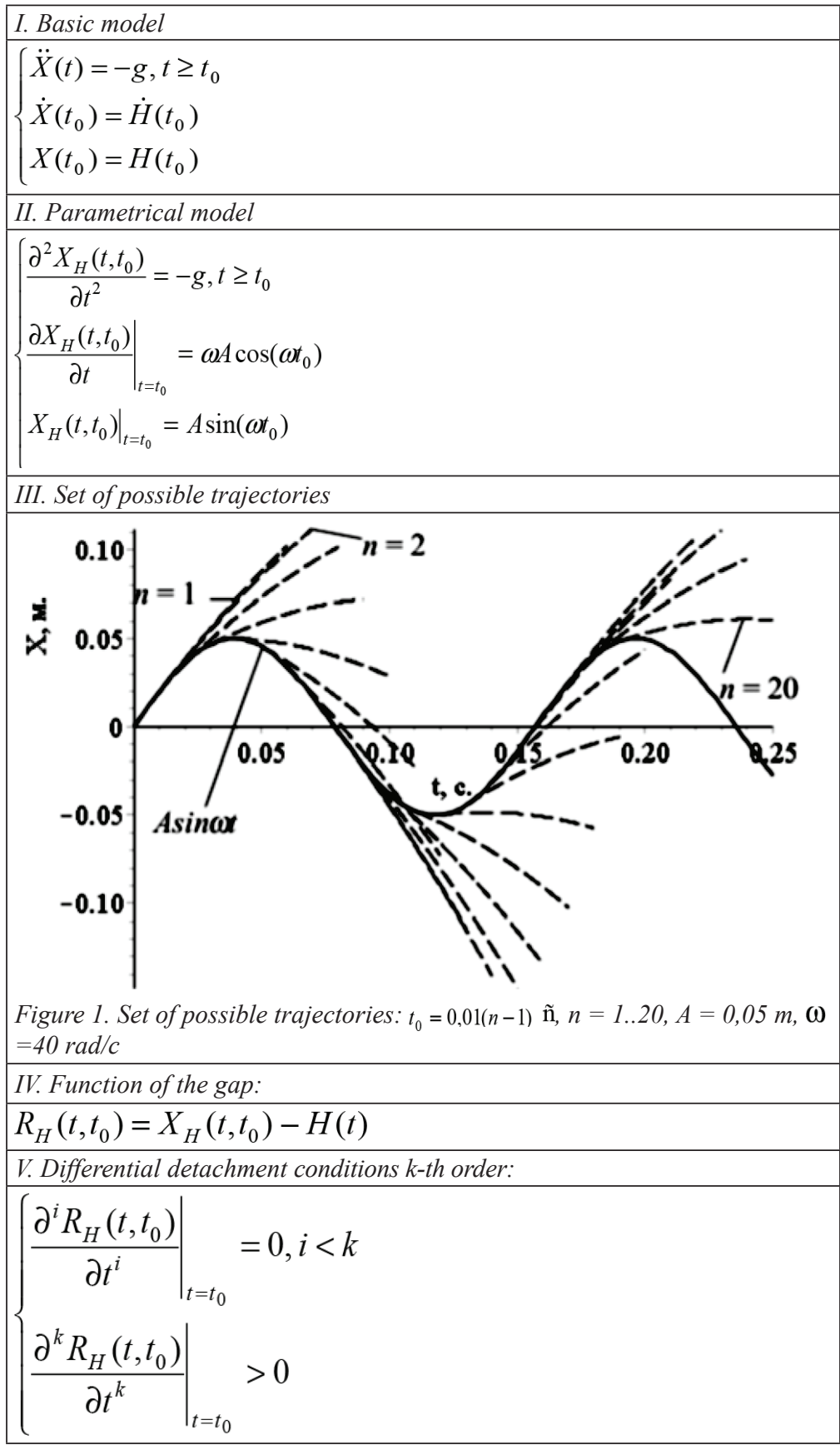


Within a concept of gap-function the separation condition of a material particle considering unilateral connections is determined by position of a particle, phase parameters of harmonious process, certain ratios between position, speed, acceleration, the sharpness (understood as the third derivative of shift by analogy with concept of smoothness or sharpness of the movements used in problems of dynamics of transport devices).

In work the approach is developed witch connected with ideas of harmonic oscillations. This approach can be extended to complex forms of movements and contacts.

The validity of application and using of function of a gap is based on theorems of necessary and sufficient conditions of detachment. The concept of an «order of detachment point» is devised to solve tasks of the comparative analysis of possibility of interactions also. This concept provides certain conveniences in an assessment of possible options and forms of the movement of particles. $\mathrm{N}$ - th an «order of detachment point» is defined by equality of the zero first $\mathrm{N}-1$ derivatives of function of a gap and positivity of its $\mathrm{N}$-th of a derivative. At a harmonious form of the movement of a surface the difference between points of a separation of the second and third order is transformed to different types of trajectories. This is because the realization of trajectories of the movement in a free phase with continuous tossing of a particle, the frequency rates of time of flight and the period of fluctuations which are dropping out of conditions, in the subsequent movements cannot be repeated a trajectory of the initial movement. In tab. 2 detachment conditions are given in terms of gap-function for points of a detachment of the second and third orders.

Table 2. Condition for function of a gape in point of second and third detachment orders

\begin{tabular}{|c|c|}
\hline point of second order of detachment & point of third order of detachment \\
\hline$\left.R\left(t, t_{0}\right)\right|_{t=t_{0}}=0$ & $\left.R\left(t, t_{0}\right)\right|_{t=t_{0}}=0$ \\
\hline$\left.R_{t}^{\prime}\left(t, t_{0}\right)\right|_{t=t_{0}}=0$ & $\left.R_{t}^{\prime}\left(t, t_{0}\right)\right|_{t=t_{0}}=0$ \\
\hline$\left.R_{t^{2}}^{\prime \prime}\left(t, t_{0}\right)\right|_{t=t_{0}}>0$ & $\left.R_{t^{2}}^{\prime \prime}\left(t, t_{0}\right)\right|_{t=t_{0}}=0$ \\
\hline- & $\left.R_{t^{3}}^{\prime \prime \prime}\left(t, t_{0}\right)\right|_{t=t_{0}}>0$ \\
\hline
\end{tabular}

In that specific case, for the harmonious law of movement of a surface it may be reasonable to consider the second and third order of a point detachment only. The physical sense of concept of an «order of a point detachment» is also essential to features of parities of elements of a set of kinematic parameters of movement.

In tab. 3 the conditions of a separation concretized for base model are presented. In fig. 2 trajectories of movement of material particles from points of a separation of the second and third usages are presented.

More complex movements with a detachment within this work have not considered yet, but they are possible and they are defined by the law of the periodic movement of a surface and features of the operating additional forces. The detailed ideas of vibration interactions received on the basis of classification of conditions of a separation of the 2nd and 3rd orders are shown in differentiation of forms of continuous tossing with a gap and impacts that predetermines interest in an assessment of height of trajectory of a particle over a surface and to opportunities of realization of the multiple modes in problems of synthesis of special sets of trajectories, etc. 
Table 3. Detachment conditions

\begin{tabular}{|c|c|}
\hline Detachment order & Analytical conditions \\
\hline 0 & - \\
\hline 1 & - \\
\hline 2 & $\frac{A \omega^{2}}{g} \sin (\omega t)>1$ \\
\hline 3 & $\left\{\begin{array}{l}\frac{A \omega^{2}}{g} \sin (\omega t)=1 \\
\cos (\omega t)>0\end{array}\right.$ \\
\hline$>3$ & Terms are not compatible \\
\hline
\end{tabular}

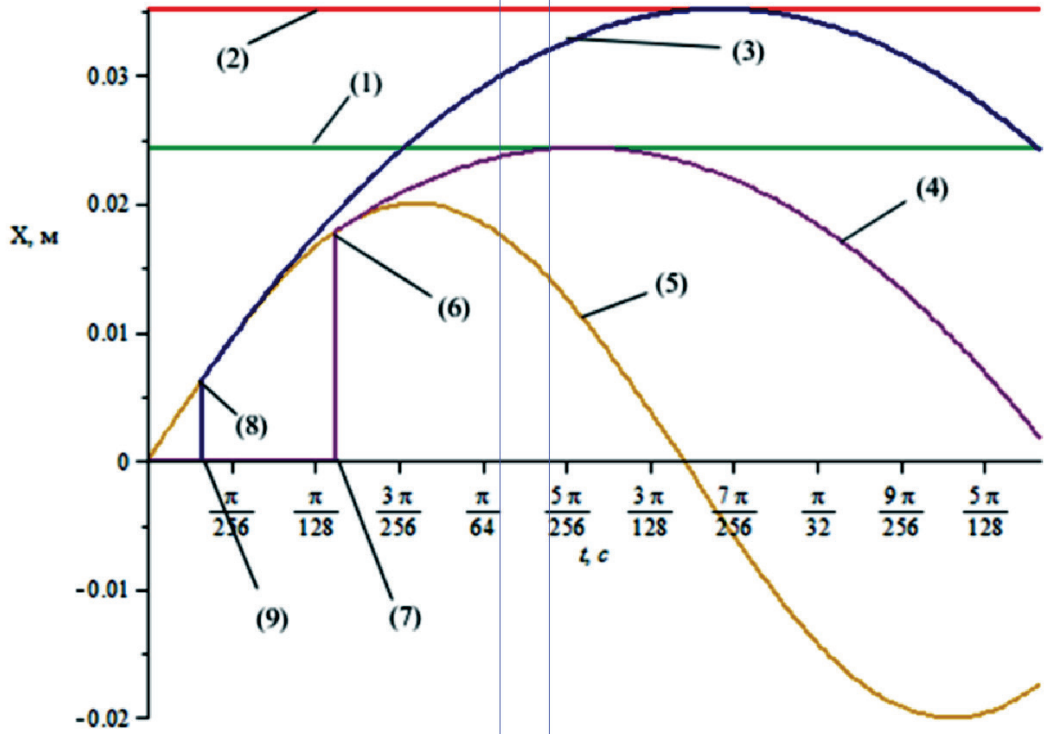

Figure 2. Trajectories of flight from points of a detachment of the second and third order: $A=0,02 \mathrm{~m}, \omega=40$ is rad/s; 5-vibration surface; 9-a timepoint of a detachment of a trajectory 3 in a point of the 8 detachment of the third order; 7 - a timepoint of a detachment of a trajectory 4 in a point 6 of the detachment of the second order; 1, 2 - the maximum altitudes of trajectories

Analytical expressions for characteristics of trajectories of the movement of particles after a detachment in timepoints of the second and third order are received on basis of using of gap-function and detachment-conditions. The regularities of formation of a trajectory with continuous tossing - the modes with the period of free approach which duration is multiple to the period of vibration of the contacting surface are investigated due to generalized approach.

The following analytical ratios of a number of key characteristics are received: conditions of a detachment of material particles from a vibration surface, estimation of free movement duration, dependence on constant additional forces and the arising resistance forces from a working environment. The role of frequency rate of the period of free flight in formation of properties of the modes with continuous tossing, etc. is established.

In fig. 3 and fig. 4 a revealed dependences are presented. 


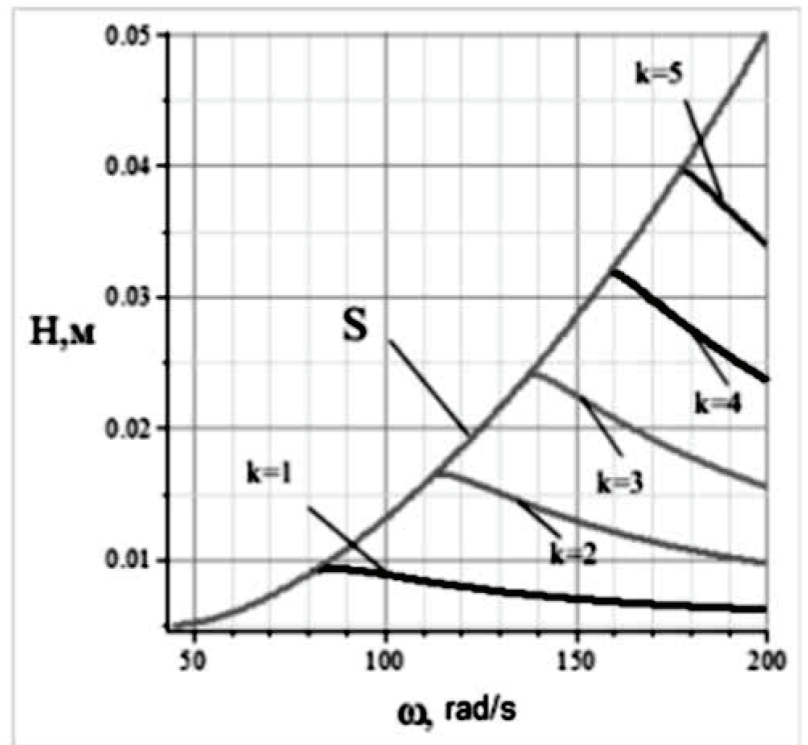

Figure 3. Height for points of the second and third order at realization of the mode of tossing in one contact: curve $S$ - height of approach of a particle from area of a detachment of the third order depending on fluctuation frequency with the fixed amplitude of fluctuation of $A=5 \mathrm{~mm}$; curve $k=1, \ldots, k=5$ - charts of heights of trajectories of particles at a detachment from area of the second order

In particular, the forms of analytical dependences of height of trajectory of a particle and a phase of a detachment of particles respectively from frequency and amplitude of fluctuations received by means of analytical expressions, reflecting connection between the multiple modes of tossing from points of a detachment of the second and third orders are presented.

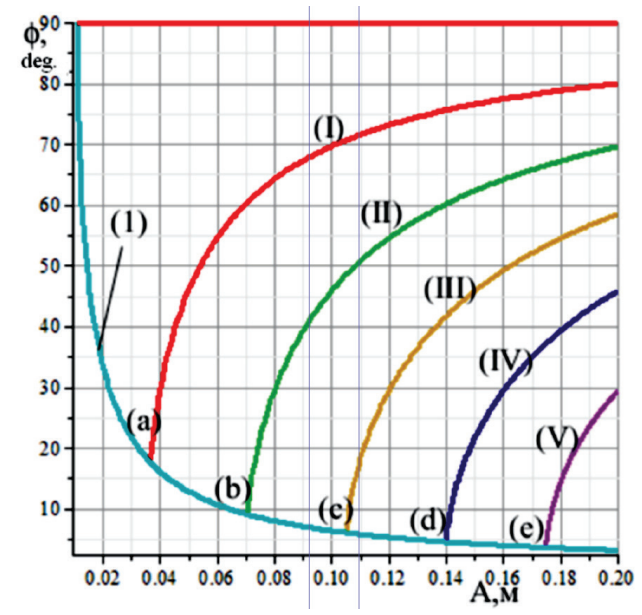

Figure 4. Phases of the modes with throwing through a certain amount of the periods: a curve $1-\mathrm{a}$ separation phase in degrees for a fluctuation surface with a frequency of 30 it is rad/c; a-e points - a phase in degrees for providing a detachment with throwing through 1-5 periods; schedules I-V - phases of detachment from points of the second order with throwing through 1-5 periods of vibration of a surface 
Analytical approach on the basis of function of a gap is used for determination of dependences of characteristics of the modes on powers of viscous friction for expanded mathematical model. Features of formation of the multiple modes of tossing in the presence of powers of viscous friction are revealed $[2,9]$.

Following from the received analytical ratios that at introduction of additional forces a big variety of the modes for use in development of technological appendices [8] can be synthesized.

Function of a gap considering with differential conditions of a detachment represents analytical reflection of all possible modes (in the framework of this mathematical model) of interaction of a material particle with the vibrating surface. The set of such conditions is a basis of development of criteria for a choice and an assessment of trajectories of the movement of material particles.

Use of function of a gap and criteria of an assessment of possible forms and features of trajectories of the movement of material particles taking into account conditions of interactions with environment represents the generalized approach allowing a pursue search development in problems of dynamic synthesis of rational vibration technologies $[13,17,12,16]$.

\section{DEFINITIONS OF CONDITIONS OF PRESERVATION OF CONTACT}

General concepts about gapless interactions between components of mechanical oscillatory system with elastic connections, including with a support on the vibrating surface, are developed [5].

As components not material points, but solid bodies of the final sizes having a contact surface, perpendicular to the direction of the movement are considered.

The mathematical model from two components (fig. 5) with use of elastic connections with the vibrating basic surface is considered. Fig. 5 shows a composite solid body which is connected with the vibrating surface by a spring $k_{1}$. The elements have only one degree of freedom and «not-holding» contact. Thus between components possibly connection violation.

The connection violation condition (emergence of a gap) is defined by parameters of the mass-inertia elements and stiffness ties. Analytical forms of the boundary ratios defining such movements at which vibration of the basis does not lead to connection violation are received. The concept about full, static and dynamic reaction of ties is entered.

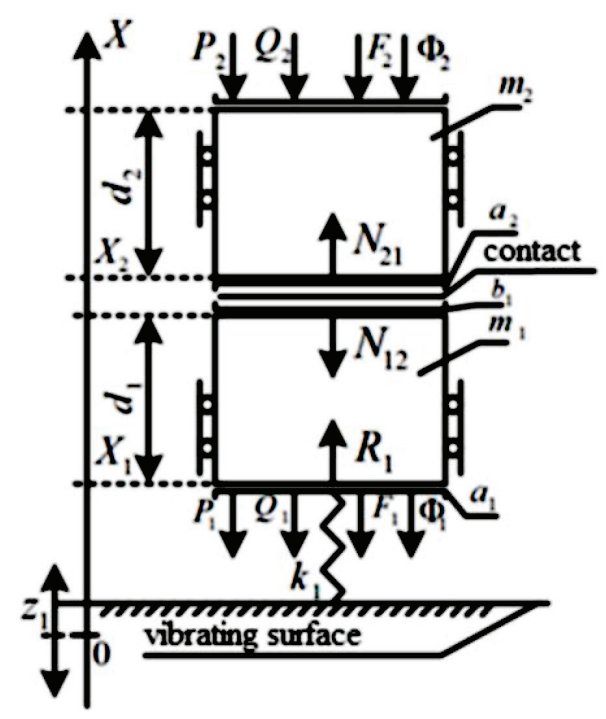

Figure 5. A compound solid body on the elastic fluctuating basis $Z_{1}: P_{i}$-powers of viscous friction; $Q_{i}-$ gravity; $F_{i}-$ constant forces; $N_{12}, N_{21}-$ full contact reactions, $\boldsymbol{k}_{1}$-stiffness coefficients

In fig. 6 schedules of dependences of amplitude of fluctuation of dynamic reaction (line 1-4) at various parameters of fluctuation of a basic surface are by way of illustration provided in comparison with static reaction (line 5). 


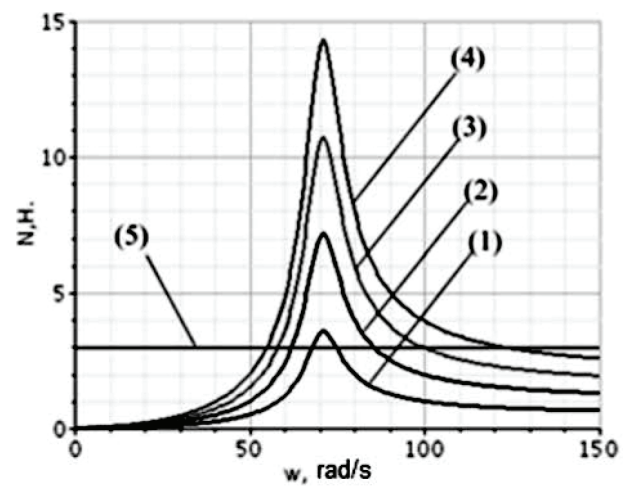

Figure 6. Estimates of amplitude of fluctuation dynamic reaction components: 1, 2, 3, 4-estimates dynamic components for amplitudes of kinematic indignation $A_{1}$ from 1 to $4 \mathrm{~mm}$; 5 -level of static reaction

Depending on parameters of mechanical system the condition of preservation of the gapless movement reads:

$$
\begin{aligned}
& \sqrt{\frac{\left(m_{2} k_{1} \omega_{1}^{2}\right)^{2}+\left(p_{2} k_{1} \omega_{1}\right)^{2}}{\left(k_{1}-\left(m_{1}+m_{2}\right) \omega_{1}^{2}\right)^{2}+\left(\left(p_{1}+p_{2}\right) \omega_{1}\right)^{2}}} \times \\
& \times A_{1}<m_{2} g+f_{c},
\end{aligned}
$$

where $p_{i}$ - coefficients of viscous friction, $k_{i}$ - stiffness coefficients, $f_{c}$ - constant force, $m_{i}$ - mass of elements, $\omega_{1}, A_{1^{-}}$are frequency and amplitude of oscillating surface.

In turn, the inequality (2) can be interpreted in the form of a set of points of parameters in amplitude-frequency area of gapless connection. In fig. 7 the line of level $\mathrm{G}$ breaks the plane of parameters $\left(A_{1}, \omega_{1}\right)$ into two areas: I - area of the gapless movement, II - area of possible formation of a gap.

Sufficient conditions of gapless vibrations of components of mechanical oscillatory system are found in the set mode. It is shown that as factors of the gapless movement of system can be considered not only frequencies and amplitudes, but also ratios of mass of components, stiffness of elastic connections, coefficients of viscous friction and external forces [16].

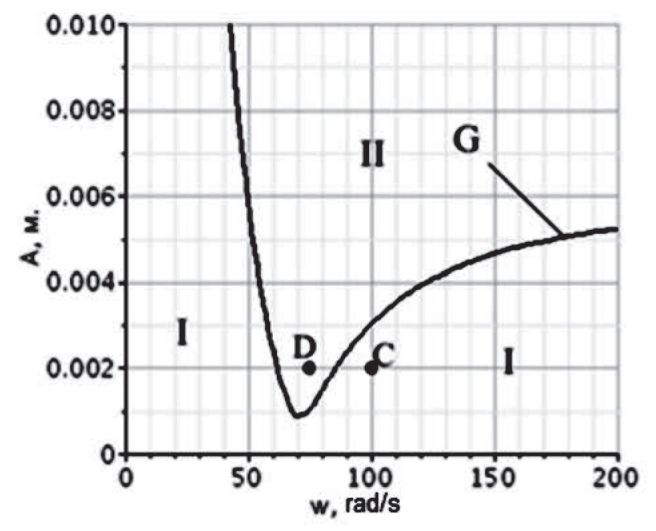

Figure 7. Amplitude-frequency area of contact: Icontact area; II - area of a possible gap; $G$ - the line of level $N_{21}^{\Sigma}$ for function $A_{1} \cdot A\left(\omega_{1}\right)$; $C$ - contact; $D$ - possible gap

Splitting amplitude-frequency area into subareas of various modes of gapless fluctuations of components depending on the additional constant force is presented in fig. 8. Constant force is considered as an external factor taking into account the mass of components [4].

The presented system of actions, rules of creation of mathematical models and analytical ratios can be considered as the developed method.

More complex challenge presented by multidimensional mathematical model is shown in fig. 9.

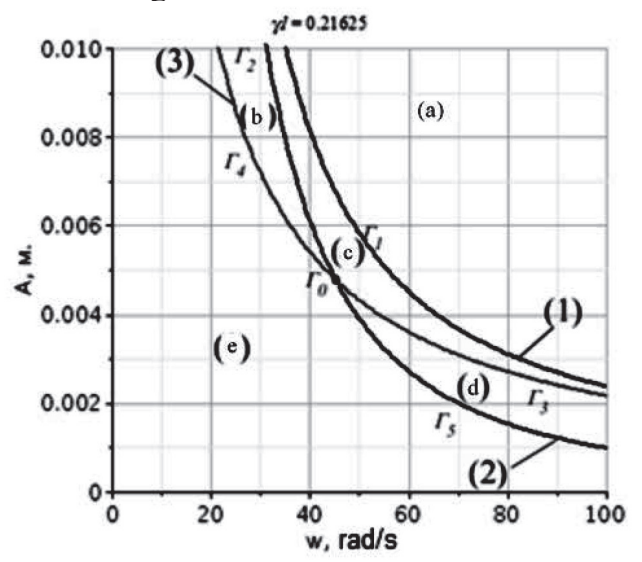

Figure 8. Domains of the characteristic modes of interaction of a solid body with a vibration surface 
At action of vibrations from basic surfaces in such structures from several components united by «not holding» connections the exception of possibility of gaps is important enough.

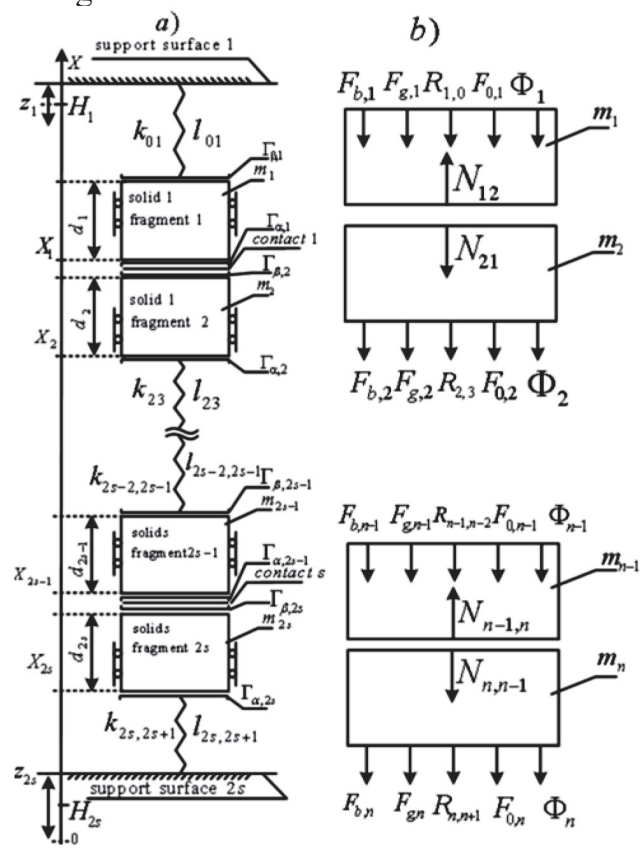

Figure 9. A compounded solid body with $S$ contacts and 2 s fragments: a) constructive scheme: $\Gamma_{\beta, i}, \Gamma_{\alpha, i}$-contact surfaces of fragments, $Z_{1}, Z_{2 s}$ - coordinates of surfaces of fluctuation, $m_{2 q-p}, m_{2 q}$-fragments of $q$-th compound solid

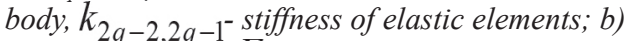
considered forces: $F_{b} ;$ - powers of viscous friction, $F_{g, i^{-}}$gravity, $F_{0, i}$ constant forces, $R_{2 q-1,2 q-2}$ , $R_{2 q, 2 q+1}$ reaction from elastic elements, $\boldsymbol{\Phi}_{i}-$ inertia forces, $i=1 . n ; N_{2 q, 2 q-1}, N_{2 q-1,2 q}$-full contact reactions, basic surfaces 1 and 2 s are vibrating, $q=1 . s, n=2 s$

Static shifts of components and reaction of connections are received in an analytical form:

$$
\left\{\begin{array}{l}
\Delta \vec{X}^{\Sigma}=\left[K_{v}-\frac{1}{2} K^{*} U U^{*} K\right]^{-1} \times \\
\times\left(V^{*} \Delta \vec{S}-K^{*} \vec{F}+\frac{1}{2} K^{*} U U^{*} \vec{F}\right) \\
\vec{N}^{\Sigma}=-\frac{1}{2} U^{*} \vec{F}-\frac{1}{2} U^{*} K\left[K_{v}-\frac{1}{2} K^{*} U U^{*} K\right]^{-1} \times \\
\times\left(V^{*} \Delta \vec{S}-K^{*} \vec{F}+\frac{1}{2} K^{*} U U^{*} \vec{F}\right)
\end{array}\right.
$$

Dynamic displacement and corresponding reactions are found in a kind:

$$
\left\{\begin{array}{l}
\overline{\vec{X}}^{\Delta}=\left[K_{v}(p)-\frac{1}{2} K(p)^{*} U U^{*} K(p)\right]^{-1} \times \\
\times\left(-K(p)^{*}+\frac{1}{2} K(p)^{*} U U^{*}\right) \vec{k}_{f} \bar{Z}, \\
\overline{\vec{N}}^{\Delta}= \\
=\left(\begin{array}{l}
-\frac{1}{2} U^{*}-\frac{1}{2} U^{*} K(p)\left[K_{v}(p)-\frac{1}{2} K(p)^{*} U \times\right. \\
\left.\times U^{*} K(p)\right]^{-1}\left(-K(p)^{*}+\frac{1}{2} K(p)^{*} U U^{*}\right)
\end{array}\right) \times \\
\times \vec{k}_{f} \bar{Z}
\end{array}\right.
$$

where $p$ - a complex variable, $\underline{\bar{Z}}$ - the image $Z$ at Laplase transformation, $\overline{\vec{X}}^{\Delta}$ and $\overrightarrow{\vec{N}}^{\Delta}$. images of dynamic displacement and dynamic reactions, $K, V, U, K_{V}(p)$ - matrixes of factors, $\Delta \vec{S}, \vec{F}, \vec{k}_{f}-$ vectors of displacement, forces and stiffness, characterizing system.

It is shown that for a multidimensional case a sufficient condition of gapless movement is the following inequality:

$$
A_{1}<\min _{q}\left\{\frac{N_{q}^{\Sigma}}{A_{(q)}\left(\omega_{1}\right)}\right\} .
$$

The right part is interpreted as function which to each frequency of fluctuation of basic surfaces puts in conformity a minimum from $s$ values which restrict an amplitude of fluctuation of corresponding surfaces.

It is shown that the offered method in case of definition of conditions gapless movements of mechanical system from several components is reduced to control of a minimum of set of the functions characterizing each connection separately.

\section{SOME TECHNICAL APPLICATIONS}

Possibilities of technical appendices of theoretical workings out [6] are considered. The sensor containing a piezo is developed. This sensor registers change of reaction of communication between the compound elements of mechanical system in the course of work of the vibrating technological machine. 
Occurrence of detachments of an inertial element with the subsequent impact is a signs of characteristic of vibrating technological process.

Technical applicability and realization of the sensor is proved by theoretical results and modeling experiment.

The domain of parameters providing a detachment of a material point from a surface is defined by a condition: $A \omega^{2}>g, A, \omega$ amplitude and frequency of fluctuation of a surface. For acknowledgement of possibility to obtain a technical result the experimental prototype of sensor and laboratory vibrating machine are developed [1].

The general contour of registration is presented in fig. 10. Experienced prototype of vibration shaker 1 is displayed on fig. 10 with container 5 filled with a mixture of steel balls. The contour is based on vibro-diagnostic systems «IDS Kamerton-D», entering into the complete set of vibration measuring equipment «MAK-8».

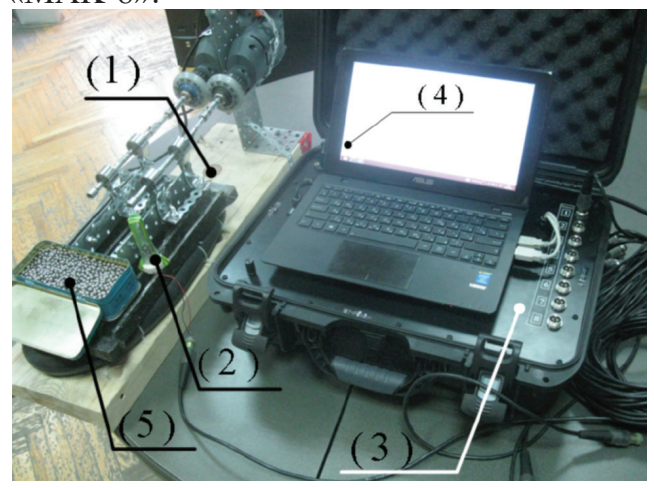

Figure 10. The general contour of registration of signals: 1 - experimental vibro-station; 2 - the sensor of registration of critical parameters; 3 - equipment for registration of signals from the sensor; 4 -output equipment of signals; 5 - the container with steel balls

The example of characteristic forms of the signals arriving from the sensor in a mode with tossing of a working environment from steel balls, is presented in fig. 11.

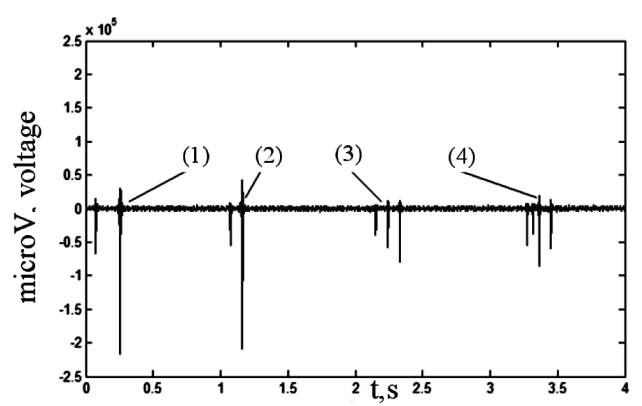

Figure 11. A characteristic signal of registration of a critical mode with tossing. (1)-(4)-forms of signals from sensors

Thus, the operation of the vibrostand in the mode of tossing of a working environment is distinctly shown through formation of the characteristic signals from the sensor registered by the equipment.

The complex of the questions connected with a substantiation of a choice of parameters is considered.

The mathematical model of vibrating machine which represents a firm body with 6 degrees of freedom, placed on elastic pneumatic elements is offered. The formation of a vibrating field is provided inertial vibrating exciter. The kinematic scheme is resulted in fig. 12.

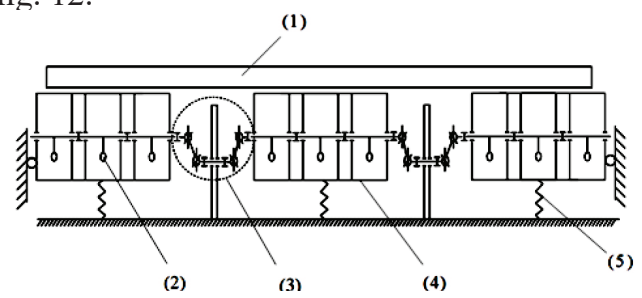

Figure 12. The kinematic scheme of vibrating machine: 1 - the container with a product; 2 - the vibrator; 3 - driveline shunt between shaft of the vibrator and shaft of synchronizer ; 4 - section of vibrating machine; 5 - an elastic element

To formation of a one-dimensional field the limiters and an emphasis are included to a design of the vibrating machine.

The estimation of a vibration condition of a technological machine is made on the basis of a set of the vibration-measuring equipment 
BY-8 (Baikal-8). One consists of station of record of signals, systems of communications, a set of sensors of electromagnetic type and the software for signals processing.

The made experiments showed sufficient coincidence to settlement data. In fig. 13 the oscillogram of characteristics of the movement of points of the working body forming a onedimensional vibration field is shown.

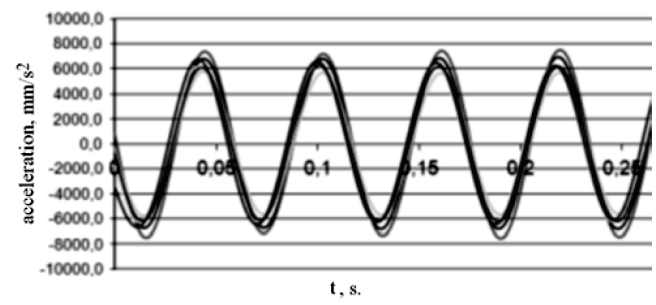

Figure 13. Schedules of oscillograms of accelerations on the basis of signals from 6 sensors

\section{CONCLUSIONS}

Let's note that the conducted researches allow to develop a certain theoretical basis in problems of an assessment of formation of families of trajectories of the movement of the material particles satisfying to such criteria as existence of multiple ratios between time of approach and the period of fluctuation of a surface, influence of deviations in separation opportunities in the necessary phase, instability of a trajectory at a separation taking into account action of additional external factors, etc.

It is shown that stability of work in certain technological modes demands control and an assessment of structure of a vibration field that is connected with certain constructive and technical features of vibration machines.

At the same time the theoretical calculations executed on models of the movement of separate particles can be used for determination of key parameters of the movement of a layer with sufficient care that require to use the special sensors fixing necessary forms of vibration interactions for setting processes.
The matrix of mutual correlation with an accuracy of several percent reflects mutual correlation of signals of sensors.

At the same time experiment is indicated that steady process of continuous tossing is characterized by existence of a pulsebeat. The frequency of pulsebeat is defined by action of two vibration factors which are formed at vibration interactions of a working environment with the vibrating surface with manifestation of processes of a partial gapless movement.

By results of experiment a number of recommendations about increase of efficiency of reliability of work of a technological complex is developed.

Thus, the features of dynamic interactions of elements of the technological complexes realizing vibration technologies with unilateral connections are studied; analytical approaches in an assessment of opportunities of vibration technological processes with continuous tossing of a working environment are defined and developed. The generalized approach is developed for problems of dynamic synthesis of the vibration technologies realized in the modes of continuous tossing. A basis of approach is introduction of concept about the function of a gap allowing to prove creation of trajectories of movements of material particles according to the certain criteria reflecting properties of trajectories of the movement of material particles in interaction with the vibrating surface.

On the basis of analytical researches and numerical modeling it is established that dependence of contact interaction on viscous friction, rigidity of elastic elements and mass of fragments of solid bodies is defined by the frequency of fluctuation of basic surfaces. 
Depending on the frequency of oscillation the variation of the generalized parameters can promote contact or lead to violation of conditions of contact. These parameters characterize the generalized strength, stiffness and weight of the fragments of the elements of the system. It is established also that depending on the frequency of fluctuation there are ranges of the mass inertia factor of violation interaction in contact $[11,3,14,7$, 15]. Influence of rigidity on contact interaction is defined by the frequency of fluctuation of basic surfaces.

\section{REFERENCES}

1. Artyunin A.I., Eliseev S. V., Kaimov E.V., Eliseev A.V. Patent Russian Federation 148250. (2014). The sensor of determination of boundary parameters of interaction of bodies in vibrating systems / Bulletin No. 33. Russia.

2. Eliseev S.V., Eliseev A.V. (2013) Determination of the coefficient of viscous friction mode multiple flip of a particle in a model problem with unilateral constraints. Systems. Methods. Technology. Bratsk. Russia No. 1, pp. 22-27.

3. Eliseev S.V., Eliseev A.V. (2014) Dynamic contact in a compounded solid taking into account «not holding» ties. Irkutsk state transport university. - Irkutsk, Dep. VINITI 27.02.2014 No. 61.Moscow. Russia.

4. Eliseev A.V. (2013) Features of interaction of a material particle with the vibrating surface depending on additional force with «not holding» ties. International magazine of applied and fundamental researches. Russia. No. 3, pp. 9-15.

5. Eliseev A.V. (2014) Technology of properties estimation of dynamic interactions in contacts of compound solid bodie. Scientific problems of transport of Siberia and the Far East. Russia. No. 1-2, pp. 179-183.

6. Eliseev A.V., Eliseev S.V., Pnev A.G., Kashuba V.B., Sitov I.S. . (2014) «Not holding» ties in dynamic interactions of the granular environment and the vibrating surface: scientific and methodological justification of technology of vibration hardening. Systems. Methods. Technologies. Bratsk. Russia. No. 3, pp. 17-31.

7. Eliseev A.V., Selvinsky V.V., Eliseev S.V. (2015) Dynamics of vibration interactions of elements of technological systems taking into account «not holding» ties: monograph.. Russia.Novosibirsk: Science. p. 332.

8. Eliseev S. V., Eliseev A.V. (2013) Features of emergence of a gap in mechanical system with «not holding» ties at pulse influence. Modern technologies. System analysis. Modeling. Irkutsk. Russia. No. 2, pp. 36-45.

9. Eliseev S. V., Eliseev A.V. (2013) Research of interaction of a material particle with the vibrating surface in the presence of power of viscous friction in a model task with «not holding» ties. Modern technologies. System analysis. Modeling. Irkutsk. Russia. No. 1, pp. 69-77.

10. Eliseev S.V., Eliseev A.V. (2012) The modes of tossing of a material particle on the vibrating surface in a model task with «not holding» connections. Modern technologies. System analysis. Modeling. Irkutsk. Russia. No. 3, pp. 86-96.

11. Eliseev S.V., Eliseev A.V. (2014) Conditions of contact fluctuations of compound solid bodies at dynamic loadings taking into account «not holding» ties. Irkutsk state transport university - Irkutsk. - Dep.VINITI 27.01.2014, No. 31. Moscow. Russia.

12. Eliseev S.V., Eliseev A.V. (2013) Methodological bases of definition of contact static and dynamic reactions taking into account «not holding» ties. Irkutsk state transport university. - Irkutsk, - Dep. VINITI 16.12.13, No. 369. Moscow. Russia.

13. Eliseev S.V., Eliseev A.V. (2013) The generalized approaches in problems of definition of contact reactions in solid bodies at static loadings taking into account «not holding» ties. Modern technologies. System analysis. Modeling. Irkutsk. Russia.. No. 4, pp 51-60.

14. Eliseev S.V., Eliseev A.V. (2014). Conditions of dynamic contact in a compound solid 
body taking into account «not holding» ties depending on parameters. Dep. VINITI 25.02.2014, No.56. Moscow. Russia.

15. Eliseev S.V., Eliseev A.V. (2015) Mathematical models of dynamics of vibration interactions of elements of technological systems taking into account «not holding» ties. Irkutsk state transport university. Dep. VINITI 21.01.2015. No. 14. Moscow. Russia.

16. Eliseev S.V., Eliseev A.V. Definition of contact reactions in compound solid bodies at dynamic loadings taking into account «not holding» ties. Modern technologies. System analysis. Modeling. Irkutsk. Russia. No. 1, (2014), pp. 45-54.

17. Eliseev S.V., Eliseev A.V., Kashuba V. B. (2013) Features of an assessment of a gap in a model problem of tossing of a material particle with «not holding» ties. Mechanical engineering and safety of vital activity. Russia. No. 1, pp. 50-56.

18. Kopylov Y.R. (2011) The dynamics of the processes of hardening vibro-impact: monograph. Russia. Voronezh: CPI «Science Book», pp. 568.

19. Panovko G.Y. (2006) Dynamics of vibrating processe. Moscow-Izhevsk: NITs «Regular and Chaotic Dynamics», Institute of Computing Technology, pp. 176.

20. Sitov I.S., Eliseev A.V. (2012) Theoretical basis of processes of interaction of a particle with a vibrating surface with unilateral constraints. Systems. Methods. Technology. Bratsk. Russia. No. 4, pp. 19-29. 